\title{
ON HARMONIC CONTINUATION
}

\author{
by M. S. P. EASTHAM
}

(Received 3 May, 1962)

1. Let $D$ be a bounded, closed, simply-connected domain whose boundary $C$ consists of a finite number of analytic Jordan curves. Let $\gamma$ be any analytic arc of $C$. Then we shall prove the following theorem.

THEOREM 1. Let $u(x, y)$ be harmonic in the interior of $D$ and continuous on $\gamma$, and let $\partial u(x, y) / \partial n=g(s)$ when $(x, y)$ is on $\gamma$, where $g(s)$ is an analytic function of arc-length $s$ along $\gamma$. Then $u(x, y)$ can be harmonically continued across $\gamma$.

Here, $\partial / \partial n$ denotes differentiation along the inner normal. A similar result for the case in which $u(x, y)$ is analytic on $\gamma$ is known [3, pp. 220-3]. The proof of Theorem 1 is given in $\S 2-4$, and an extension to the case in which $D$ is a more general region is given in $\S 5$.

2. First let $v(r, \theta)$ be any function which is harmonic in $r<R, 0<\theta<\pi$, and continuous in the closure of this region, $r, \theta$ being polar coordinates. Also, let $\partial v(r, \theta) / \partial y$ tend to zero as $y \rightarrow 0$, when $-R<x<R$. Define $v(\theta)$ by $v(\theta)=v(R, \theta)$ if $0 \leqq \theta \leqq \pi$, and $v(\theta)=v(R, 2 \pi-\theta)$ if $\pi \leqq \theta \leqq 2 \pi$. Then it is proved in [4] (with a different notation) that the function

$$
V(r, \theta)=\frac{R^{2}-r^{2}}{2 \pi} \int_{0}^{2 \pi} \frac{v(\phi)}{R^{2}+r^{2}-2 R r \cos (\theta-\phi)} d \phi,
$$

which is harmonic in $r<R$ and continuous in $r \leqq R$, is equal to $v(r, \theta)$ in $r \leqq R, 0 \leqq \theta \leqq \pi$. Thus $V(r, \theta)$ is a harmonic continuation of $v(r, \theta)$ into the lower half of the region $r \leqq R$.

3. Next, let $w(x, y)$ be harmonic in $x^{2}+y^{2}<1, y>0$ and continuous in $x^{2}+y^{2}<1, y \geqq 0$, and let $\partial w(x, y) / \partial y$ tend to $g(x)$ as $y \rightarrow 0$ when $-1<x<1$, where $g(x)$ is analytic in $-1<x<1$.

If $-1<a<1$, we have

$$
g(x)=\sum_{n=0}^{\infty} c_{n}(x-a)^{n},
$$

the expansion being valid in some neighbourhood of $a$. Then the series $\sum c_{n}(z-a)^{n}$, where $z=x+i y$, represents an analytic function of $z, g(z)$ say, in some region $|z-a| \leqq R$, and we can take $R$ to satisfy $-1<a-R<a+R<1$.

$$
\text { Let } \quad G(z)=\sum_{n=0}^{\infty} \frac{c_{n}(z-a)^{n+1}}{n+1},
$$

and let $W(x, y)=\operatorname{im} G(z)$. Then $G(z)$ is analytic in $|z-a| \leqq R$, and so $W(x, y)$ is harmonic there. Also, 


$$
\frac{\partial W(x, y)}{\partial y}=\frac{\partial}{\partial x}\{\operatorname{re} G(z)\}
$$

and the right-hand side is $g(x)$ when $y=0$ and $a-R \leqq x \leqq a+R$. Hence, by $\S 2, w(x, y)-$ $W(x, y)$ can be harmonically continued across the segment $y=0, a-R \leqq x \leqq a+R$. But $W(x, y)$ is harmonic in the whole region $|z-a| \leqq R$ and so, by varying $a$, we obtain a harmonic continuation of $w(x, y)$ across the $x$-axis, $-1 \leqq x \leqq 1$.

4. We are now in a position to establish Theorem 1 . We can transform the interior of $D$ conformally into the region $x_{1}^{2}+y_{1}^{2}<1, y_{1}>0$ in the $z_{1}$-plane, where $z_{1}=x_{1}+i y_{1}$, in such a way that $\gamma$ becomes the diameter $y_{1}=0,-1 \leqq x_{1} \leqq 1$, the transformation being $z=f\left(z_{1}\right)$, say. Then the transformation is conformal in a domain which extends outside $D$ across the arc $\gamma$ [2, p. 186]. Furthermore, $g(s)$ is transformed into an analytic function of $x_{1}$.

Let $u(x, y)$ be transformed into the function $u_{1}\left(x_{1}, y_{1}\right)$. Then we have the relation between the normal derivatives

$$
\left[\frac{\partial u_{1}\left(x_{1}, y_{1}\right)}{\partial y_{1}}\right]_{y_{1}=0}=\left|f^{\prime}\left(x_{1}\right)\right| \frac{\partial u(x, y)}{\partial n}
$$

for $(x, y)$ on $\gamma$, where $\left|f^{\prime}\left(x_{1}\right)\right|$ denotes $\left[\left|f^{\prime}\left(z_{1}\right)\right|\right]_{y_{1}=0}$, which can be shown to be an analytic function of $x_{1}$. Since now the right-hand side of (1) is an analytic function of $x_{1}$, it follows from $\S 3$ that $u_{1}\left(x_{1}, y_{1}\right)$ can be harmonically continued across the segment $y_{1}=0,-1 \leqq x_{1} \leqq 1$. On transforming back to the $(x, y)$-plane, we obtain the result that $u(x, y)$ can be harmonically continued across the arc $\gamma$.

5. Suppose for the moment that $\gamma$ is any analytic Jordan arc. Then there is a region $D^{\prime}$ containing $\gamma$ the points of which can be said to be on one side or the other of $\gamma$ [1, pp. 192-3]. Let $D_{+}^{\prime}$ denote the subregion consisting of points on one side of $\gamma$ and $D_{-}^{\prime}$ the subregion consisting of points on the other side of $\gamma$, the points of $\gamma$ belonging to both $D_{+}^{\prime}$ and $D_{-}^{\prime}$. We can now obtain the extension of Theorem 1.

THEOREM 2. Let $D$ be any region for which there is an analytic Jordan arc $\gamma$ with the property that if $P$ is any point of $\gamma$ then there is a neighbourhood $N(P)$ of $P$ such that $N(P) \cap D=N(P) \cap D_{+}^{\prime}$. Let $u(x, y)$ satisfy the same conditions as in Theorem 1 with respect to $D$ and $\gamma$. Then $u(x, y)$ can be harmonically continued across $\gamma$.

It is clearly possible to construct a subregion of $D, D_{1}$ say, which is bounded, closed, simply-connected, and whose boundary consists of $\gamma$ (or any finite part of $\gamma$ if $\gamma$ extends to infinity) and a finite number of analytic Jordan arcs. Indeed,

$$
D_{1} \subset \underset{P \in \gamma}{\bigcup}\{N(P) \cap D\}
$$

Also, $u(x, y)$ is harmonic in the interior of $D_{1}$. It follows from Theorem 1 that $u(x, y)$ can be harmonically continued across $\gamma$, and this is the required result. 


\section{REFERENCES}

1. L. V. Ahlfors, Complex analysis (New York, 1953).

2. Z. Nehari, Conformal mapping (New York, 1952). 1946).

3. W. J. Sternberg and T. L. Smith, The theory of potential and spherical harmonics (Toronto,

4. T. Ugaeri, On the harmonic prolongation, J. Math. Soc. Japan 1 (1949), 262-5.

\section{Merton College}

OXFORD 